\title{
Errata* $^{*}$
}

\section{Resonance Vol.24, No.7, 2019}

How to Design Experiments in Animal Behaviour

4. How Do Bees Estimate the Distance Flown?

(DOI: https://doi.org/10.1007/s12045-019-0836-y)

On page 749, Line no. 17

“....total of $\mathbf{6}$ meters..." to be read as "....total of $\mathbf{1 2}$ meters..."

*DOI: https://doi.org/10.1007/s12045-019-0854-9 